REVISTA MATEMÁTICA COMPLUTENSE

Volumen 11, número 1: 1998

http://dx.doi.org/10.5209/rev_REMA.1998.v11.n1.17323

\title{
On the relative Nash approximation of analytic maps.
}

\author{
Alessandro TANCREDI and Alberto TOGNOLI
}

\begin{abstract}
Let $X, Y$ be real or complex Nash spaces and $Z$ a subspace of $X$; the paper deals with the approximation of analytic maps $\phi: X \longrightarrow Y$, Nash on $Z$, with Nash maps $\psi: X \longrightarrow Y$ such that $\left.\psi\right|_{Z}=\left.\phi\right|_{Z}$.
\end{abstract}

\section{Introduction}

The problem of the approximation of analytic objects by algebraic ones has been considered by quite a number of mathematicians ([BCR], [DLS], [Sh], [TT1 ], [TT2], [T2]) and it is one of the main and oldest problem in the Nash geometry where cohomological methods are not available. A strong limit of the approximation methods is that they work mainly for the non-singular spaces.

Recently the use of the so-called Néron desingularization ([Pol, $[\mathrm{Sp}])$, a deep result of commutative algebra, has allowed a significant development in the approximation methods in the real case ([CRS]) and in the complex case ([Le]).

Let $X, Y$ be Nash spaces and $Z$ a subspace of $X$; we deal with the approximation of analytic maps $\phi: X \longrightarrow Y$, Nash on $Z$, with Nash maps $\psi: X \longrightarrow Y$ such that $\left.\psi\right|_{Z}=\phi \mid z$. In the past we had given some results in the non-singular case ([TT1], [TT2]) and now, using

1991 Mathematics Subject Classification: 32C07, 58A07, 14P20.

Servicio Publicaciones Univ. Complutense. Madrid, 1998. 
the results of [CRS] and [Le], we can consider the singular real case (2.3, $2.5,2.6)$ and the singular complex case $(4.6,4.7)$.

Moreover we point out the unicity, up to analytic isomorphisms, of the Nash structure of a coherent compact real analytic space. This suggests the study of Nash structures of some couples of spaces that we begin in this paper with quite different methods, unfortunately available only in the non-singular case (3.2).

For the complex Nash spaces, that will be examined more carefully in a forthcoming paper, we can obtain here weaker results $(4.6,4.7$, 4.8). The complex Nash functions are indeed less plastic than real ones and the results that are available in the complex case $(4.2,4.3)$ are less satisfactory than in the real one (1.1).

\section{Preliminary remarks and definitions}

Let $k$ be the field of real numbers $\mathbb{R}$ or the field of complex numbers $\mathbb{C}$.

We will consider the strong topology on the algebraic subvarieties of $k^{n}$.

Let $D$ be an open domain in $k^{n}$; an analytic function $f: D \longrightarrow k$ is called a Nash function if there exists a non-zero polynomial $P \in$ $k\left[t_{1}, \ldots, t_{n+1}\right]$ such that $P(x, f(x))=0$ for every $x \in D$. A map $f$ : $D \longrightarrow k^{q}$ is called a Nash map if every component of $f$ is a Nash function. The composition of Nash maps is a Nash map and the inverse function theorem holds for Nash maps. We denote by $\mathcal{N}_{k^{n}}$ the sheaf of Nash functions on $k^{n}$ : it is a coherent subsheaf of the sheaf $\mathcal{O}_{k^{n}}$ of analytic functions on $k^{n}$.

A Nash (resp. analytic) subspace $X$ of $k^{n}$ is a locally closed subset that is, locally, the zero set of a finite number of Nash (resp. analytic) functions. Nash (resp. analytic) functions on open sets of $X$ are locally restrictions of Nash (resp. analytic) functions on open sets of $k^{n}$. We denote by $\mathcal{N}_{X}$ (resp. $\mathcal{O}_{X}$ ) the sheaf of Nash (resp. analytic) functions on $X$.

A Nash (resp. analytic) space is a $k$-ringed space that is locally isomorphic to Nash (resp. analytic) subspaces of $k^{n}$. We recall that a Nash space is (locally) irreducible if and only if it is (locally) irreducible as an analytic space. A Nash manifold is a Nash space that is smooth as an analytic space. 
We speak of real functions (spaces) for $k=\mathbb{R}$ and of complex functions (spaces) for $k=\boldsymbol{C}$.

We say that a real Nash space is coherent if it is coherent as an analytic space. All the analytic spaces we consider are paracompact with bounded embedding dimension.

A Nash space $X$ is normal if the ring $\mathcal{N}_{X, x}$ is integrally closed in its full ring of quotients for every $x \in X ; X$ is normal as a Nash space if and only if it is normal as analytic space.

We will use the following deep results of [CRS] (Theorem 1.6).

Theorem 1.1. Let $X$ be a coherent compact real Nash subspace of $\mathbb{R}^{n}$.

i) The ideal $\mathcal{I}_{X} \subset \mathcal{N}_{\mathbb{R}^{n}}$ of Nash functions vanishing on $X$ is generated by finitely many global Nash functions.

ii) Every Nash function on $X$ extends to $\mathbb{R}^{n}$.

We recall that a diffeotopy $\theta$ on a differentiable manifold $X$ is a differentiable map $\theta: X \times[0,1] \longrightarrow X$ such that the map $x \longmapsto \theta(x, t)$ is a diffeomorphism of $X$ for every $t \in[0,1]$.

A time-dependent vector field on $X$ is a differentiable map $\chi$ from $X \times[0,1]$ to the tangent bundle $T(X)$ such that $\chi(x, t) \in T_{x}(X)$ for every $x \in X, t \in[0,1]$. If we denote by $\tau$ the canonical projection $X \times[0,1] \longrightarrow X$ it is easy to see that the time dependent vector fields on $X$ can be identified with the differentiable sections of the pullback vector bundle $\tau^{*} T(X)$.

Theorem 1.2. There exists a one to one correspondence between time dependent vector fields $\chi$ on a compact differentiable manifold $X$ and diffeotopies $\theta$ on $X$ given by

$$
\frac{\partial \theta}{\partial t}(x, t)=\chi(\theta(x, t), t) .
$$

Proof. See [Hi]

Let $E$ be a differentiable vector bundle on $X$ and $Z$ a subset of $X$. We say that a continuous section $t$ of $E$ on $Z$ is a differentiable section if for every $x \in Z$ there exist an open neighborhood $U$ of $x$ in $X$ and a differentiable section $s: U \longrightarrow E$ such that $\left.s\right|_{Z \cap U}=\left.t\right|_{Z \cap U}$. 
Lemma 1.3. Let $X$ be a differentiable manifold, $Z$ a closed subset of $X, E$ a differentiable vector bundle on $X$. Any differentiable section $t: Z \longrightarrow E$ extends to $X$.

Proof. By a straightforward use of a partition of unity argument.

Let $\left(S_{i}\right)_{i \in I}$ be a finite family of closed differentiable submanifolds of $X$. We say that the submanifolds $S_{i}$ are in general position if for all subsets $\left\{i_{1}, \ldots, i_{r}\right\},\left\{j_{1}, \ldots, j_{s}\right\}$ of distinct elements of $I$ the submanifolds $S_{i_{1}} \cap \cdots \cap S_{i_{r}}$ and $S_{j_{1}} \cap \cdots \cap S_{j_{s}}$ intersect transversally.

The following result generalizes the theorem of extension of diffeotopies ([Hi])).

Theorem 1.4. Let $X$ be a compact differentiable manifold, $\left(S_{i}\right)_{i \in I}$ a finite family of closed differentiable submanifolds of $X$ in general position and let $\left(\theta_{i}\right)_{i \in I}$ be a family of diffeotopies $\theta_{i}: S_{i} \times[0,1] \longrightarrow S_{i}$ such that $\theta_{i}=\theta_{j}$ on $\left(S_{i} \cap S_{j}\right) \times[0,1]$ for every $i, j \in I$. There exists a diffeotopy $\theta: X \times[0,1] \longrightarrow X$ such that $\left.\theta\right|_{S_{i} \times[0,1]}=\theta_{i}$ for every $i \in J$.

Proof. By 1.2 it is enough to prove that if $E$ is a differentiable vector bundle on $X$ and $\left(s_{i}\right)_{i \in I}$ a family of differentiable sections $s_{i}$ of $E$ on $S_{i}$ such that $s_{i}\left|S_{i} \cap S_{j}=s_{j}\right| S_{i} \cap S_{j}$ for every $i, j \in I$, then there exists a differentiable section $s$ of $E$ on $X$ such that $s \mid s_{i}=s_{i}$ for every $i \in I$. Let us consider the continuous section $t$ of $E$ on $\cup S_{i}$ defined by $\left.t\right|_{S_{i}}=s_{i}$ for every $i \in I$. By $1.3 \mathrm{it}$. is enough to prove that the section $t$ is differentiable, i.e. that it is locally restriction of differentiable sections on open subsets of $X$. Since the question is local we can suppose that the $s_{i}$ are differentiable functions and the conclusion follows easily by the transversality condition.

\section{Real Nash approximation}

Let $X, Y$ be two real analytic spaces. We will consider on the space $\mathcal{E}(X, Y)$ (resp. $\mathcal{O}(X, Y))$ of differentiable (resp. analytic) maps from $X$ to $Y$ the Whitney $C^{1}$ topology (see e.g. [GMT]) and by approximation of maps between real analytic spaces we will mean always approximation in this topology. Let $\left(U_{\dot{q}}\right)_{i \in I}$ be a locally finite open covering of $X$ and $\left(V_{i}\right)_{i \in I}$ a locally finite open covering of $Y$ such that there exist locally closed analytic embeddings $\phi_{i}: U_{i} \longrightarrow \mathbb{R}^{n_{i}}, \psi_{i}: V_{i} \longrightarrow \mathbb{R}^{p_{i}}$ for every $i \in I$; let $\left(K_{i}\right)_{i \in I}$ be a covering of $X$ such that $K_{i}$ is a compact subset 
of $U_{i}$ for every $i \in I$ and $\left(\epsilon_{i}\right)_{i \in I}$ a family of positive real numbers. Let $\theta_{0}: X \longrightarrow Y$ be a differentiable map and let us consider the sets

$$
\begin{aligned}
\mathcal{U} & =\mathcal{U}\left(\theta_{0},\left(U_{i}, \phi_{i}\right)_{i \in I},\left(V_{i}, \psi_{i}\right)_{i \in I},\left(K_{i}\right)_{i \in I},\left(\epsilon_{i}\right)_{i \in I}\right) \\
& =\left\{\theta \in \mathcal{E}(X, Y) \mid \theta\left(K_{i}\right) \subset V_{i},\left\|\psi_{i} \theta \phi_{i}^{-1}-\psi_{i} \theta_{0} \phi_{i}^{-1}\right\|_{\phi_{i}\left(K_{i}\right)}^{1}<\epsilon_{i} \forall i \in I\right\}
\end{aligned}
$$

where \|\|$_{\phi_{i}\left(K_{i}\right)}^{1}$ is the $C^{1}$-norm on $\phi_{i}\left(K_{i}\right)$. The Whitney topology on $\mathcal{E}(X, Y)$ (resp. $\mathcal{O}(X, Y))$ is the topology that has as a base all possible sets $\mathcal{U}$.

Theorem 2.1. Let $X, Y$ be two coherent real analytic spaces. The set of isomorphisms is open in $\mathcal{O}(X, Y)$.

Proof. We follow the method of the proof given in [Hi] for differentiable manifolds. Let $\theta_{0}$ be an isomorphism and $\mathcal{U}$ a neighborhood of $\theta_{0}$ in the Whitney topology. If $H$ is a compact set in $Y$ there exists a finite number of compact $K_{i}$ that covers $\theta^{-1}(H)$ for every analytic map $\theta: X \longrightarrow Y$ such that $\theta\left(K_{i}\right) \subset V_{i}$. It follows that we can find a neighborhood $\mathcal{U}$ of $\theta_{0}$ such that every $\theta \in \mathcal{U}$ is a proper map. On the other hand if $\mathcal{U}$ is small enough every $\theta \in \mathcal{U}$ is a local embedding of analytic spaces. Moreover since $\theta_{0}\left(K_{i}\right) \cap \theta_{0}\left(X-U_{i}\right)=\emptyset$ for every $i \in I$ we can find $\mathcal{U}$ in such a way that $\theta\left(K_{i}\right) \cap \theta\left(X-U_{i}\right)=\emptyset$ and $\left.\theta\right|_{U_{i}}$ is injective for all $\theta \in \mathcal{U}, i \in I$. Therefore, for $\mathcal{U}$ small enough, every $\theta \in \mathcal{U}$ is a closed embedding. Since $X$ and $Y$ are coherent there exist decompositions into irreducible components, $X=\cup_{\nu \in \mathbb{N}} X^{\nu}, Y=\cup_{\nu \in \mathbb{N}} Y^{\nu}$, and we can suppose that $\theta_{0}\left(X^{\nu}\right)=Y^{\nu}$ for every $\nu \in \mathbb{N}$. Moreover, since $\theta\left(X^{\nu}\right)$ is a closed irreducible subset of $Y$, if $\theta$ is near enough to $\theta_{0}$, we have $\theta\left(X^{\nu}\right)=Y^{\nu}$ for every $\nu \in \mathbb{N}$ and then $\theta$ is an isomorphism.

Corollary 2.2. Let $X, Y$ be two coherent real Nash spaces. The set of isomorphisms is open in the space $\mathcal{N}(X, Y)$ of Nash maps from $X$ to $Y$ with the topology induced by the topology of $\mathcal{O}(X, Y)$.

Proof. A Nash map that is an analytic isomorphism is a Nash isomorphism.

Theorem 2.3. Let $X$ be a coherent real Nash space and $Z$ a closed Nash subspace of $X$. The following conditions are equivalent.

i) Let $Y$ be a real Nash space and $F: X \times Y \longrightarrow \mathbb{R}^{q}$ a Nash map. Every analytic map $\phi: X \longrightarrow Y$ Nash on $Z$ that satisfies the 
equation $F(x, \phi(x))=0$ on $X$ can be approximated by a Nash map $\psi: X \longrightarrow Y$ such that $\left.\psi\right|_{Z}=\left.\phi\right|_{Z}$ and $F(x, \psi(x))=0$ for every $x \in X$.

ii) Let $Y$ be a real Nash space and $F: Y \longrightarrow \boldsymbol{R}^{q}$ a Nash map. Every analytic map $\phi: X \longrightarrow Y$ Nash on $Z$ that satisfies the equation $F(\phi(x))=0$ on $X$ can be approximated by a Nash map $\psi: X \longrightarrow$ $Y$ such that $\left.\psi\right|_{Z}=\left.\phi\right|_{Z}$ and $F(\psi(x))=0$ for every $x \in X$.

iii) Let $Y$ be a real Nash space and $\phi: X \longrightarrow Y$ an analytic map Nash on $Z$. Then $\phi$ can be approximated by a Nash map $\psi: X \longrightarrow Y$ such that $\left.\psi\right|_{Z}=\phi \mid z$.

Proof. The implications $i) \Rightarrow i i)$ and $i i) \Rightarrow i i i$ ) are obvious.

iii) $\Rightarrow$ i) Let $\Gamma=\{(x, y) \in X \times Y \mid F(x, y)=0\}$ and let us consider the analytic map $\theta: X \longrightarrow \Gamma$ defined by $x \mapsto(x, \phi(x))$. There exists a Nash map $\omega: X \longrightarrow \Gamma$ arbitrarily near to $\theta$ such that $\left.\omega\right|_{Z}=\left.\theta\right|_{Z}$. Let $\pi: \Gamma \longrightarrow X$ and $\tau: \Gamma \longrightarrow Y$ be the Nash maps induced by the canonical projections and $\rho=\pi \omega$. If $\omega$ is near enough to $\theta$ the map $\rho$ is near to the identity of $X$ and then by $2.2 \rho$ can be supposed a Nash isomorphism. It follows that the map $\psi=\tau \omega \rho^{-1}$ satisfies the required conditions.

Remark 2.4. In [CRS] it is proved that the equivalent conditions of the previous theorem hold for $Z=\emptyset$ when $X$ is a compact coherent subspace of $\mathbb{R}^{n}$ and $Y$ is a subspace of $\mathbb{R}^{p}$ zero set of finitely many Nash functions on $\mathbb{R}^{p}$.

We point out that the previous theorem is quite formal and it can be stated in any situation, in the differentiable, analytic or Nash setting, where the set of isomorphisms is open in some suitable topology.

However we remark that, if $Y$ is singular, differentiable maps are not approximable by analytic ones as we can see by a simple example: let $X=\mathbb{R}$, or even $X=\mathbb{P}(\mathbb{R}), Y=\left\{x \in \mathbb{R}^{2} \mid x_{1}^{3}+x_{1}^{2}-x_{2}^{2}=0\right\}$ and let us take two $C^{\infty}$ bump functions $\lambda, \mu: \mathbb{R} \longrightarrow[0,1]$ such that $\operatorname{supp}(\lambda)=[-1,0]$ and $\operatorname{supp}(\mu)=[0,1]$. It is easy to check that the differentiable map $\phi: X \longrightarrow Y$ defined by $\phi(x)=(\lambda(x)+\mu(x),(\lambda(x)-$ $\left.\mu(x))(1+\lambda(x)+\mu(x))^{\frac{1}{2}}\right)$ for every $x \in X$ is not approximable by any analytic map since for every neighborhood $U$ of 0 in $X \phi(U)$ is never 
contained in only one analy tic branch through the origin of the algebraic curve.

Theorem 2.5. Let $X \subset \mathbb{R}^{n}$ be a compact coherent real Nash space, $Z$ a closed coherent Nash subspace of $X, H: \mathbb{R}^{p} \longrightarrow \mathbb{R}^{t}, F: X \times \mathbb{R}^{p} \longrightarrow \mathbb{R}^{q}$ Nash maps and $Y=\left\{y \in \mathbb{R}^{p} \mid H(y)=(0, \ldots, 0)\right\}$. Every analytic map $\phi: X \longrightarrow Y$ Nash on $Z$ that satisfies the equation $F(x, \phi(x))=0$ on $X$ can be approximated by a Nash map $\psi: X \longrightarrow Y$ such that $\left.\psi\right|_{Z}=\left.\phi\right|_{Z}$ and $F(x, \psi(x))=0$ for every $x \in X$.

Proof. By considering the Nash map $\tilde{F}: X \times \mathbb{R}^{p} \longrightarrow \mathbb{R}^{q+t}$ defined by $\tilde{F}(x, y)=(F(x, y), H(y))$ we can reduce to the case $Y=\mathbb{R}^{p}$.

Let $\phi=\left(\phi_{1}, \ldots, \phi_{p}\right)$; we will reduce to the case $\phi_{j} \mid z=0$ for every $j=1, \ldots, p$. By 1.1 there exist Nash functions $g_{j} \in \mathcal{N}_{X}(X)$ such that $\left.g_{j}\right|_{z}=\left.\phi_{j}\right|_{z}$ for every $j=1, \ldots, p$. Let us consider the Nash map $F^{\prime}: X \times \mathbb{R}^{p} \longrightarrow \mathbb{R}^{q}$ defined by $F^{\prime}(x, y)=F\left(x, y_{1}+g_{1}(x), \ldots, y_{p}+g_{p}(x)\right)$ and the analytic map $\phi^{\prime}: X \longrightarrow \mathbb{R}^{p}$ where $\phi_{j}^{\prime}=\phi_{j}-g_{j}$ for every $j=1, \ldots, p$. Let us suppose that there exists a Nash map $\psi^{\prime}: X \longrightarrow \mathbb{R}^{p}$ arbitrarily near to $\phi^{\prime}$ such that $\psi_{j}^{\prime}(x)=0$ for every $x \in Z, j=1, \ldots, p$ and $F^{\prime}\left(x, \psi^{\prime}(x)\right)=0$ for every $x \in X$. Let us consider the Nash map $\psi: X \longrightarrow \boldsymbol{R}^{p}$ defined by $\psi_{j}=\psi_{j}^{\prime}+g_{j}$ for every $j=1, \ldots, p$ : the map $\psi$ is arbitrarily near to $\phi$ and obviously $\left.\psi\right|_{Z}=\phi \mid z$; moreover for every $x \in X$ we have $F(x, \psi(x))=F\left(x, \psi_{1}^{\prime}(x)+g_{1}(x), \ldots \psi_{p}^{\prime}(x)+g_{p}(x)\right)=$ $F^{\prime}\left(x, \psi^{\prime}(x)\right)=0$.

Let $\mathcal{I}_{Z}$ be the ideal of $\mathcal{N}_{X}$ of the Nash functions vanishing on $Z$; since $\mathcal{I}_{Z}$ is coherent by 1.1 it is generated as $\mathcal{N}_{X}$-module by finiteiy many Nash functions $h_{i} \in \mathcal{N}_{X}(X), i=1, \ldots, t$. Let $\mathcal{J}_{Z}$ be the ideal of $\mathcal{O}_{X}$ of the analytic functions vanishing on $Z$; we have $\mathcal{I}_{Z} \mathcal{O}_{X}=\mathcal{J}_{Z}$ ([BCR]) and then $\mathcal{J}_{Z}$ is generated by $h_{1}, \ldots, h_{t}$ as $\mathcal{O}_{X}$-module.

Let $G: X \times \mathbb{R}^{p} \times \mathbb{R}^{p t} \longrightarrow \mathbb{R}^{q+p}$ be the Nash map defined by

$$
\begin{aligned}
& G_{j}(x, y, z)=F_{j}(x, y) j=1, \ldots, q, \\
& G_{q+l}(x, y, z)=y_{l}-\sum_{i=1}^{t} z_{i l} h_{i}(x) l=1, \ldots, p .
\end{aligned}
$$

By the Theorem B of H. Cartan for real analytic spaces ([Ca]) there exist analytic functions $\alpha_{i l} \in \mathcal{O}_{X}(X), i=1, \ldots, t, l=1, \ldots, p$ such that $\phi_{l}=\sum_{i=1}^{t} \alpha_{i l} h_{i}$. 'Then the analytic map $\theta: X \longrightarrow \mathbb{R}^{p} \times \mathbb{R}^{p t}$ defined by $\theta(x)=\left(\phi(x), \alpha_{11}(x), \ldots, \alpha_{t p}(x)\right)$ satisfies the equation $G(x, \theta(x))=0$ on $X$. By the Remark 2.4 there exists a Nash map $\omega: X \longrightarrow \mathbb{R}^{p} \times \mathbb{R}^{p t}$ 
arbitrarily near to $\theta$ such that $G(x, \omega(x))=0$ for every $x \in X$. If $\pi$ is the canonical projection $\mathbb{R}^{p} \times \mathbb{R}^{p t} \longrightarrow \mathbb{R}^{p}$ the Nash map $\psi=\pi \omega$ satisfies the required conditions.

Corollary 2.6. Let $X \subset \mathbb{R}^{n}$ be a compact coherent real Nash space, $Z \subset X$ a closed coherent Nash subspace, $H: \mathbb{R}^{p} \longrightarrow \mathbb{R}^{t}$ a Nash map and $Y=\left\{y \in \mathbb{R}^{p} \mid H(y)=(0, \ldots, 0)\right\}$. For any analytic map $\phi: X \longrightarrow Y$ Nash on $Z$ there exists a Nash map $\psi: X \longrightarrow Y$ arbitrarily near to $\phi$ such that $\psi_{\left.\right|_{Z}}=\left.\phi\right|_{Z}$. Moreover

i) if $\phi$ is an analytic isomorphism $\psi$ is a Nash isomorphism;

ii) if $\phi$ is a section of a Nash map $\pi: Y \longrightarrow X$ and the approximation is good enough then $\psi$ is a section of $\pi$.

Proof. The main assertion is contained in 2.5 .

i) It follows from 2.2 .

ii) If $\theta$ is a Nash map near enough to $\phi$, with $\left.\theta\right|_{Z}=\phi \mid z$, the map $\rho=\theta \pi$ is near to the identity and by 2.2 can be supposed a Nash isomorphism. It follows that the Nash map $\psi=\theta \rho^{-1}$ is a section of $\pi$ near to $\phi$ with $\left.\psi\right|_{Z}=\phi Z$.

In the smooth case the previous result can be easily improved.

Corollary 2.7. Let $X \subset \mathbb{R}^{n}, Y \subset \mathbb{R}^{p}$ be two compact real Nash manifolds and $Z$ a closed coherent Nash subspace of $X$. For any differentiable map $\phi: X \longrightarrow Y$ Nash on $Z$ there exists a Nash map $\psi: X \longrightarrow Y$ arbitrarily near to $\phi$ such that $\left.\psi\right|_{Z}=\phi \mid z$. Moreover

i) if $\phi$ is a diffeomorphism $\psi$ is a Nash isomorphism;

ii) if $\phi$ is a section of a Nash map $\pi: Y \longrightarrow X$ and the approximation is good enough then $\psi$ is a section of $\pi$.

Proof. By 2.6 it is enough to find $\psi$ analytic. By the existence of the analytic tubular neighborhood of $Y$ in $\mathbb{R}^{p}$ the proof follows from a result on the relative approximation of differentiable functions: for any differentiable function $f: X \longrightarrow \mathbb{R}$ such that $\left.f\right|_{Z}$ is analytic there exists an analytic function $g: X \longrightarrow \mathbb{R}$ arbitrarily near to $f$ with $\left.g\right|_{Z}=\left.f\right|_{Z}$ ([T1]) . 
Remark 2.8. From 2.6 it follows that the equivalent conditions of 2.3 hold if $Z$ is a closed coherent subspace of a compact coherent subspace $X \subset \mathbb{R}^{n}$ and $Y$ is a subspace of $\mathbb{R}^{p}$ zero set of finitely many Nash functions on $\mathbb{R}^{p}$.

By the previous results it is possible to approximate an analytic section $s$ of a Nash fiber bundle $E$ on $X$ that is Nash on a closed Nash subspace $Z$ of $X$ with a Nash section $\tilde{s}$ such that $\left.\tilde{s}\right|_{Z}=\left.s\right|_{Z}$ when the total space $E$ is the zero set of finitely many Nash functions on a numeric space. In particular if $E$ is a strongly Nash vector bundle such an approximation is well-known ([BCR], [TT3]).

\section{Unicity of structures of Nash couples}

The Corollary 2.6 states the unicity of the Nash structure on a compact real analytic space up to analytic isomorphisms and the Corollary 2.7 the unicity up to diffeomorphisms in the smooth case. They suggest versions for couples of spaces: let $X, Y$ be compact real Nash spaces, $S \subset X, T \subset Y$ closed Nash subspaces and $\phi: X \longrightarrow Y$ an analytic (resp. differentiable) isomorphism such that $\phi(S)=T$. Is there a Nash isomorphism $\psi: X \longrightarrow Y$ such that $\psi(S)=T$ ? If the spaces are coherent, $\phi$ is analytic on $X$ and Nash on $S$ the Corollary 2.6 gives an affirmative answer. In general the question is more difficult. In the following we will give a positive answer in the smooth case.

Let $X \subset \mathbb{R}^{n}$ and $Y \subset \mathbb{R}^{p}$ be two compact real Nash manifolds and $\left(S_{i}\right)_{i \in I},\left(T_{i}\right)_{i \in I}$ two finite families of closed Nash submanifolds $S_{i} \subset X$, $T_{i} \subset Y$. We say that $\left(X,\left(S_{i}\right)_{i \in I}\right),\left(Y,\left(T_{i}\right)_{i \in I}\right)$ are Nash couples if the families of submanifolds $\left(S_{i}\right)_{i \in I}$ and $\left(T_{i}\right)_{i \in I}$ are in general position. If $I$ is a singleton $\{i\}$ we write simply $S$ and $T$ instead of $S_{i}$ and $T_{i}$.

A differentiable (resp. analytic, Nash) isomorphism of such couples is a differentiable (resp. analytic, Nash) isomorphism $\phi: X \longrightarrow Y$ such that $\phi\left(S_{i}\right)=T_{i}$ for every $i \in I$.

Lemma 3.1. Let $\phi:(X, S) \longrightarrow(Y, T)$ be a differentiable isomorphism of Nash couples and let $Z$ be a closed coherent Nash subspace of $S$ such that $\left.\phi\right|_{Z}$ is Nash. There exist:

i) a Nash isomorphism $\psi: S \longrightarrow T$ arbitrarily near to $\left.\phi\right|_{S}$ such that $\left.\psi\right|_{Z}=\left.\phi\right|_{Z}$ 
ii) a diffeotopy $\theta: T \times[0,1] \longrightarrow T$ such that

$$
\begin{array}{ll}
\theta(y, 0) & =y \text { for every } y \in T \\
\theta(\phi(x), 1) & =\psi(x) \text { for every } x \in S, \\
\theta(\phi(x), t) & =\phi(x) \text { for every } x \in Z \text { and } t \in[0,1]
\end{array}
$$

iii) a Nash isomorphism of couples $\hat{\phi}:(X, S) \longrightarrow(Y, T)$ such that $\left.\hat{\phi}\right|_{Z}=\left.\phi\right|_{Z}$.

Proof. i) It follows from 2.7.

ii) Let $\pi: W \longrightarrow T$ be a Nash tubular neighborhood ([Sh]) of $T$ in $\mathbb{R}^{p}$. If $\psi$ is near enough to $\left.\phi\right|_{S}$ we can define a diffeotopy $\theta: T \times[0,1] \longrightarrow$ $T$ by putting

$$
\theta(y, t)=\pi\left(y(1-t)+\psi \phi^{-1}(y) t\right) \text { for every } y \in T \text { and } t \in[0,1] .
$$

If $x \in Z$ for every $t \in[0,1]$ we have

$$
\theta(\phi(x), t)=\pi(\phi(x)(1-t)+\psi(x) t)=\pi(\phi(x))=\phi(x) .
$$

On the other hand it is easy to see that $\theta(\phi(x), 1)=\psi(x)$ for every $x \in S$.

iii) By 1.4 there exists a diffeotopy $\eta: Y \times[0,1] \rightarrow Y$ such that $\eta(\phi(x), t)=\theta(\phi(x), t)$ for every $x \in S$ and $t \in[0,1]$. Let us denote by $\phi^{\prime}$ the diffeomorphism $X \longrightarrow Y$ defined by $x \longmapsto \eta(\phi(x), 1)$. We have $\left.\phi^{\prime}\right|_{S}=\left.\psi\right|_{S}$ and, as in $i$ ) we can find a Nash isomorphism $\hat{\phi}: X \longrightarrow Y$ such that $\left.\hat{\phi}\right|_{S}=\left.\psi\right|_{S}$. Of course $\left.\hat{\phi}\right|_{Z}=\left.\psi\right|_{Z}=\left.\phi\right|_{Z}$.

Theorem 3.2. Let $\left(X,\left(S_{i}\right)_{i \in I}\right)$ and $\left(Y,\left(T_{i}\right)_{i \in I}\right)$ be two Nash couples. If there exists a differentiable isomorphism $\phi:\left(X,\left(S_{i}\right)_{i \in I}\right) \longrightarrow\left(Y,\left(T_{i}\right)_{i \in I}\right)$, then there exists a Nash isomorphism of couples $\hat{\phi}:\left(X,\left(S_{i}\right)_{i \in I}\right) \longrightarrow$ $\left(Y,\left(T_{i}\right)_{i \in I}\right)$.

Proof. For any positive integer $m$ let us denote by $\mathcal{P}_{m}$ the set of subsets $H=\left\{i_{1}, \ldots, i_{m}\right\}$ of $I$ such that $i_{h} \neq i_{k}$ for $h \neq k$. If $H \in \mathcal{P}_{m}$ and $i \notin H$ let us denote by $H(i)$ the element $\left\{i_{1}, \ldots, i_{m}, i\right\}$ of $\mathcal{P}_{m+1}$. For any $H=\left\{i_{1}, \ldots, i_{m}\right\}$ let us denote by $S_{H}$ (resp. $T_{H}$ ) the intersection $S_{i_{1}} \cap \cdots \cap S_{i_{m}}$ (resp. $T_{i_{1}} \cap \cdots \cap T_{i_{m}}$ ). By the hypotheses made $S_{H}$ and $T_{H}$ are Nash submanifolds and $\phi\left(S_{H}\right)=T_{H}$ for every $H \in \mathcal{P}_{m}, m \in \mathbb{N}$; moreover, by the transversality conditions again, there exist $q \in \mathbb{N}$, 
$H \in \mathcal{P}_{q}$ such that $S_{H} \neq \emptyset$ and $S_{L}=\emptyset$ for every $L \in \mathcal{P}_{m}, m>q$, or $\mathcal{P}_{m}=\emptyset$.

Let $H \in \mathcal{P}_{q}$ such that $S_{H} \neq \emptyset$; by 3.1 there exist a Nash isomorphism $\psi_{H}: S_{H} \longrightarrow T_{H}$ near to $\left.\phi\right|_{S_{H}}$ and a diffeotopy $\theta_{H}: T_{H} \times[0,1] \longrightarrow T_{H}$ such that $\theta(\phi(x), 1)=\psi_{H}(x)$ for every $x \in S_{H}$.

Let $L \in \mathcal{P}_{q-1}$ and let us consider the sets $L(i)$ of $\mathcal{P}_{q}$. By 1.4 there exists a diffeotopy $\theta_{L}: T_{L} \times[0,1] \longrightarrow T_{L}$ such that $\left.\theta_{L}\right|_{T_{L(i)} \times[0,1]}=\theta_{L(i)}$ for every $i \in I$ such that $S_{L(i)} \neq \emptyset$. If $T_{L} \neq \emptyset$ and $T_{L(i)}=\emptyset$ for every $i \in I$ as above we find a diffeotopy $\theta_{L}$. It follows that for every $J, L \in \mathcal{P}_{q-1}$, with $T_{J} \neq \emptyset$ and $T_{L} \neq \emptyset$ we have $\theta_{J}=\theta_{L}$ on $\left(T_{J} \cap T_{L}\right) \times[0,1]$.

By proceeding step by step we find a diffeotopy $\theta_{H}: T_{H} \times[0,1] \longrightarrow$ $T_{H}$ for every $H \in \mathcal{P}_{m}$, with $m \leq q$ such that $\left.\theta_{H}\right|_{T_{J} \times[0,1]}=\theta_{J}$ for every $J \supset H$. By 1.4 there exists a diffeotopy $\theta_{q}: Y \times[0,1] \longrightarrow Y$ such that $\left.\theta_{q}\right|_{T_{H} \times[0,1]}=\theta_{H}$ for every $H \in \mathcal{P}_{m}, m \leq q$.

Let $\phi_{q}: X \longrightarrow Y$ be the diffeomorphism defined by $x \longmapsto \theta_{q}(\phi(x), 1)$; by the construction made $\phi_{q}$ defines a differentiable isomorphism of Nash couples $\left(X,\left(S_{i}\right)_{i \in I}\right) \longrightarrow\left(Y,\left(T_{i}\right)_{i \in I}\right)$ such that $\phi_{q} \mid S_{H}$ is a Nash isomorphism for every $H \in \mathcal{P}_{q}$.

Let us consider now $H \in \mathcal{P}_{q-1}$ such that $S_{H} \neq \emptyset$. From 3.1, applied to $\left.\phi_{q}\right|_{S_{H}}$ with $Z=\left\{U S_{J} \mid J \in \mathcal{P}_{q}, J \supset H\right\}$, it follows that there exists a Nash isomorphism $\psi_{H}: S_{H} \longrightarrow T_{H}$ such that $\psi_{H}\left|S_{J}=\phi_{q}\right| S_{J}$ for every $J \in \mathcal{P}_{q}$.

By repeating the above constructions we find a differentiable isomorphism of Nash couples $\phi_{q-1}:\left(X,\left(S_{i}\right)_{i \in I}\right) \longrightarrow\left(Y,\left(T_{i}\right)_{i \in I}\right)$ such that $\phi_{q-1} \mid S_{H}$ is a Nash isomorphism for every $H \in \mathcal{P}_{q-1}$.

After a finite number of steps we find a diffeomorphism $\phi_{1}:\left(X,\left(S_{i}\right)_{i \in I}\right) \longrightarrow\left(Y,\left(T_{i}\right)_{i \in I}\right)$ such that $\left.\phi_{1}\right|_{S_{i}}$ is Nash for every $i \in I$.

By applying 3.1 again to $\phi_{1}$ with $Z=\cup_{i \in I} S_{i}$ we get the conclusion.

\section{Complex Nash approximation}

Theorem 4.1. Let $X$ be a normal closed complex Nash subspace of an open set $A \subset \mathbb{C}^{n}$ and $f: X \longrightarrow \mathscr{P}^{p}$ a Nash map. There exist a normal affine subvariety $N \subset \mathbb{C}^{n+p+t}$, a regular map $\pi: N \longrightarrow X$ induced by the canonical projection $\mathbb{C}^{n+p+t} \longrightarrow \mathscr{C}^{n}$, an open Nash embedding. $\sigma: X \longrightarrow N$ such that $\pi \sigma=i d_{X}$ and a regular map $h: N \longrightarrow \mathscr{\sigma}^{p}$ such that $h \sigma=f$. 
Proof. It is a version of the complex Artin Mazur Theorem (see e.g. [BCR], [TT2]).

Corollary 4.2. Let $X$ be a closed normal complex Nash subspace of an open set $A \subset \mathbb{C}^{n}, f_{1}, \ldots, f_{p} \in \mathcal{N}_{X}(X)$ Nash functions and $Z=\{x \in$ $\left.X \mid f_{1}(x)=\cdots=f_{p}(x)=0\right\}$.

i) There exists an algebraic subvariety $T \subset N$ such that $\left.\sigma\right|_{Z}$ is an open embedding into $T$.

ii) The ideal sheaf $\mathcal{I}_{Z} \subset \mathcal{N}_{X}$ of the Nash functions vanishing on $Z$ is generated by finitely many Nash functions on $X$ vanishing on $Z$.

Proof. i) Let $f=\left(f_{1}, \ldots, f_{p}\right)$ and $h=\left(h_{1}, \ldots, h_{p}\right)$; the affine variety $T=\left\{z \in N \mid h_{1}(z)=\cdots=h_{p}(z)=0\right\}$ has the required properties.

ii) By i) we may assume that $Z=T \cap \Omega$ where $\Omega$ is an open set of an algebraic subvariety $N \subset \mathscr{C}^{n}$ and $T$ is an algebraic subvariety of $N$. Let $\mathcal{R}_{N}$ be the sheaf of regular functions on $N$ and $\mathcal{J}_{T} \subset \mathcal{R}_{N}$ the ideal sheaf of the regular functions vanishing on $T$. Since $\mathcal{J}_{T}$ is generated by finitely many regular functions on $N$ vanishing on $T$ it is enough to prove that $\mathcal{J}_{T} \mathcal{N}_{N}=\mathcal{I}_{T}$. We can assume $N=\mathscr{C}^{n}$ and the conclusion follows using a classical result of Chevaliey as in the analogous statement on the ideal sheaf of analytic functions vanishing on $T$ (see e. g. [Ru]) since the Nullstellensatz holds for complex Nash germs.

Theorem 4.3. Let $X$ be a normal closed complex Nash subspace of a Runge domain $A \subset \mathbb{C}^{n}$ and $K$ a holomorphically convex compact subset of $X$.

i) There exist Nash functions $f_{1}, \ldots, f_{s}$ on a polynomial polyhedron $U$ in $A$, with $K \subset U$, such that $X \cap U=\left\{z \in U \mid f_{1}(z)=\cdots=\right.$ $\left.f_{s}(z)=0\right\}$.

ii) For every Nash function $f \in \mathcal{N}_{X}(X)$ there exist a polynomial polyhedron $U \subset A$, with $K \subset U$, and $F \in \mathcal{N}_{\mathbb{C}^{n}}(U)$ such that $\left.F\right|_{U \cap X}=\left.f\right|_{U \cap X}$.

Proof. See [TT2]

Theorem 4.4. Let $K$ be a holomorphically convex compact subset of an affine algebraic variety $N, \phi$ a complex analytic map from a neighborhood of $K$ to $\mathscr{A}^{p}, G$ a complex Nash map from a neighborhood of the graph of 
$\phi$ in $N \times \mathscr{O}^{p}$ to $\mathbb{C}^{q}$ such that $G(x, \phi(x))=0$ for every $x \in K$. Then $\phi$ can be uniformly approximated on $K$ by complex Nash maps $\psi$ from a neighborhood of $K$ to $\mathbb{Q}^{p}$ such that $G(x, \psi(x))=0$ for every $x \in K$.

Proof. See [Le].

Lemma 4.5. Let $A \subset \mathscr{C}^{n}$ be a Runge domain, $X \subset A$ a normal complex closed Nash subspace, $K$ a holomorphically convex compact subset of $X$, $B \subset \mathscr{O}^{p}$ an open set, $F: X \times B \longrightarrow \mathbb{C}^{q}$ a Nash map and $\phi: X \longrightarrow B$ an analytic map such that $F(x, \phi(x))=0$ for every $x \in K$. There exist an open neighborhood $U$ of $K$ in $X$ and a Nash map $\psi: U \longrightarrow B$ arbitrarily near to $\phi$ on $K$ such that $F(x, \psi(x))=0$ for every $x \in K$.

Proof. By 4.1 there exist a normal affine subvariety $N \subset \mathbb{C}^{n+t}$ and an open Nash embedding $\sigma: X \longrightarrow N$ such that $\pi \sigma=\mathrm{id}_{X}$, where $\pi: N \longrightarrow X$ is induced by the canonical projection $\mathbb{Q}^{n+t} \longrightarrow \mathbb{C}^{n}$. Since the open set $\Omega=\sigma(X)$ of $N$ is a closed subset of $A \times \mathbb{C}^{t}$ then $\sigma(K)$ is holomorphically convex in $N$.

Let us consider the Nash map $G: \Omega \times B \longrightarrow \mathscr{C}^{q}$ defined by $G(z, y)=$ $F\left(\sigma^{-1}(z), y\right)$ and the complex analytic map $\phi \sigma^{-1}$ on a neighborhood of $\sigma(K)$.

By 4.4 there exist an open neighborhood $V$ of $\sigma(K)$ in $\Omega$ and a Nash map $\psi^{\prime}: V \longrightarrow \mathbb{C}^{q}$, arbitrarily near to $\phi \sigma^{-1}$ on $\sigma(K)$ such that $G\left(z, \psi^{\prime}(z)\right)=0$ for every $z \in \sigma(K)$. The Nash function $\psi=\psi^{\prime} \sigma$ on the open set $U=\sigma^{-1}(V)$ satisfies the condition $F(x, \phi(x))=0$ for every $x \in K$ and approximates $\phi$ on $K$; moreover if $\psi$ is near enough to $\phi$ on $K$ we can suppose $\psi(U) \subset B$.

Theorem 4.6. Let $A \subset \mathbb{C}^{n}$ a Runge domain, $B \subset \mathscr{O}^{p}$ an open set, $X \subset A$ a normal closed complex Nash subspace, $K$ a compact set in $X$, $g_{1}, \ldots, g_{q} \in \mathcal{N}_{\mathbb{C}^{p}}(B)$ Nash functions and $Y=\left\{y \in B \mid g_{1}(y)=\cdots=\right.$ $\left.g_{q}(y)=0\right\}$. Every complex analytic map $\phi$ from a neighborhood of $K$ in $X$ to $Y$ can be approximated on $K$ by a Nash map $\psi$ defined on a neighborhood $U$ of $K$. Moreover $U$ and $\psi$ can be chosen in such a way that:

i) if $\phi$ is an analytic isomorphism $\psi$ is a Nash isomorphism $U \cong$ $\psi(U)$;

ii) if $\phi$ is a section of a Nash map $\pi: Y \longrightarrow X$ then $\psi$ is a section of $\pi$ on $U$. 
Proof. By considering a neighborhood of the holomorphic hull of $K$ in $X$ we can assume $K$ holomorphically convex and taking the Nash map $F: X \times B \longrightarrow \mathbb{C}^{q}$ defined by $(x, y) \longmapsto\left(g_{1}(y), \ldots, g_{q}(y)\right)$ the existence of $\psi$ follows from 4.5 .

i) It is a standard fact (see e.g. [GMT]) that, if the approximation is strong enough, $\psi$ is an injection and a local embedding on a neighborhood of $K$; since $Y$ is locally irreducible $\psi$ is an open map ([Fi]).

ii) It is a consequence of $i$ ) as in 2.6 .

Remark 4.7. By the previous theorem it is not difficult to state a complex version, taking the approximation on a neighborhood of a compact set $K$, of the equivalent assertions of 2.3. More difficult is to check when the equivalent assertions hold. Using 4.2, 4.3 and the Theorem B of $\mathrm{H}$. Cartan for complex spaces ([Fi]) the proof of 2.5 can be repeated when $X$ and $Y$ are closed normal subspaces of Runge domains, $Z$ a closed subspace of $X$ and $K$ a compact set in $Z$.

Moreover from the results of [TT3] it is also possible to approximate analytic sections with Nash sections of Nash vector bundles generated by finitely many Nash global sections.

The proof of 2.5 can be also repeated in the setting of affine varieties as in the following theorem that was given in [TT1 $]$ for $Y=\mathbb{C}$.

Theorem 4.8. Let $X$ and $Y$ be two affine algebraic subvarieties, $Z$ an algebraic subvariety of $X, K$ a holomorphically convex compact set in $X$ and $\phi: X \longrightarrow Y$ a complex analytic map algebraic on $Z$. There exist Nash maps on open neighborhoods $U$ of $K$ in $X$, arbitrarily near to $\phi$ on $K$ such that $\left.\psi\right|_{U \cap Z}=\left.\phi\right|_{U \cap Z}$.

\section{References}

[BCR] J. Bochnak, M. Coste, M. F. Roy, Géométrie algébrique réelle, Ergebnisse der Mathematik und ihrer Grenzgebiete 3. Folge, Band 12, Springer, Berlin, Heidelberg, New York, 1987.

[Ca] H. Cartan, Variétés analytiques réelles et variétés analytiques complexes, Bull. Soc. Math. Frauce 85 (1957), 77-99.

[CRS] M. Coste, J. M. Ruiz, M. Shiota, Approximation in compact Nash manifolds, Amer. J. Math. 117 (1995), 905-927. 
[DLS] J. P. Demailly, L. Lempert, B. Shiffman, Algebraic approximation of holomorphic maps from Stein domains to projective manifolds, Duke Math. J. (1994), 333-363.

[Fi] G. Fischer, Complex analytic geometry, Lecture Notes in Mathematics 538, Springer, Berlin, Heidelberg, New York, 1976.

[GMT] F. Guaraldo, P. Macrí, A. Tancredi, Topics on real analytic spaces, Advanced Lectures in Mathematics, Vieweg, Wiesbaden, 1986.

[Hi] M. W. Hirsh, Differential topology, Graduate text in Mathematics 33, Springer, Berlin, Heidelberg, New York, 1976.

[Le] L. Lempert, Algebraic approximation in analytic geometry, Inv. Math. 121 (1995), 335-353.

[Po] D. Popescu, General Néron desingularization, Nagoya Math. J. 100 (1985), 97-126.

[Ru] M. J. Ruiz, The basic theory of power series, Advanced Lectures in Mathematics, Vieweg, Wiesbaden, 1993.

[Sh] M. Shiota, Nash manifolds, Lecture Notes in Math. 1269, Springer, Berlin, Heidelberg, New York, 1987.

[Sp] M. Spivakovsky, Smoothing of ring homomorphisms, approximation theorems and the Bass-Quillen conjecture, preprint, 1994.

[TT1 A. Tancredi, A. Tognoli, Relative approximation theorems of Stein manifolds by Nash manifolds, Boll. Un. Mat. It. 3-A (1989), 343-350.

['TT2] A. Tancredi, A. Tognoli, On the extension of Nash functions, Math. Ann. 288 (1990), 595-604.

[TT3] A. Tancredi, A. Tognoli, Some remarks on the classification of complex Nash vector bundles, Bull. Sc. Math. 117 (1993), 173-183.

[T1] A. Tognoli, Un teorema di approssimazione relativo, Atti Acc. Naz. Lincei , 54 (1973), 496-502. 
[12] A. Tognoli, Algebraic approximation of manifolds and spaces, Sém. Bourbaki, 548 (1979/80).

Dipartimento di Matematica Università di Perugia Via Vanvitelli, 1 I-06123 Perugia, Italy

e-mail: altan@unipg.it

Dipartimento di Matematica Università di Trento Via Sommarive, 14 I-38050 Povo (TN), Italy

Recibido: 25 de Septiembre de 1996 Revisado: 28 de Abril de 1997 